\title{
THE PATHOLOGY
}

OF

\section{CHRONIC BRIGHT'S DISEASE WITH CONTRACTED KIDNEY,}

WITH RSPECIAL RETEREICE TO THE

THEORY OF、“ARTERIO-CAPILLARY FIBROSIS.”

BY

GEORGE JOHNSON, M.D., F.R.S., PROFESSOR OF THE PRINCIPLES $\triangle$ ND PRACTICR OF MEDICINE IN RING's COLIEGT, LONDON, AND PHYSICIAN TO KING's COLLFG HOSPITAL.

Received December 4th-Read December 10th, 1872.

IN the fifty-first volume of the 'Medico-Chirurgical Transactions' I published a paper in which I described and figured what I look upon as a genuine hypertrophy of the muscular walls of the minute arteries from various tissues in cases of chronic Bright's disease. I there stated that I had observed a constant relation between hypertrophy of the arterial walls and hypertrophy of the left ventricle of the heart. And I gave what I believe to be the true physiological interpretation of the phenomena. I have looked upon this discovery of hypertrophy of the arterial walls as the most interesting result of a long series of observations extending over a period of rather more than a quarter of a century, and I supposed that my doctrine relating to this particular subject had met with very general assent. In this belief, however, I now find that 
I was mistaken. The recently issued volume of the 'Transactions' contains a very elaborate paper by Sir Wm. Gull and Dr. Sutton in which the authors, while giving me some credit for having directed attention to thickening of the arteries, dissent entirely from my interpretation of the phenomena, and propound doctrines of great novelty and interest relating to the pathology of Bright's disease. In the present communication, I propose to inquire whether these novel doctrines are consistent with known pathological facts and established physiological principles.

The authors give a brief summary of the conclusions at which they have arrived at pp. 295-6 of their paper. The chief novelty to which they lay claim as a discovery is the doctrine that a "hyalin-fibroid" change in the minute arteries and capillaries in various tissues is the primary and essential phenomenon in cases of chronic Bright's disease with contracted kidney. This degeneration of the blood-vessels, they say, commonly begins in the kidney but it may begin in other organs; so that hypertrophy of the heart with degeneration of the blood-vessels may be found associated with healthy kidneys, the contraction and atrophy of the kidney when present being but "part and parcel of the general morbid change."

The morbid state commonly called chronic Bright's disease with contracted kidney is believed by Sir Wm. Gull, and Dr. Sutton to be essentially an "arterio-capillary fibrosis," the morbid changes being allied to senile changes, but " probably due to distinct causes not yet ascertained." Before proceeding to examine their description of the hyaliu-fibroid change in the blood-vessels and in the kidneys, I desire to direct attention to some difficulties which have been suggested to me by a perusal of their paper.

In order to prove that the cardiac and vascular changes are not a result of the kidney disease they refer, at p. 286 (vol. lv), to three cases in which the kidneys were "little contracted" but the minute arteries and capillaries were much thickened by " hyalin-fibroid" substance. On turning to these cases I find that case 7, at p. 300, was that of a man, 
forty-two years of age who had very emphysematous lungs, with capillary bronchitis; case 10 , at p. 303 , was sixty-nine years of age and died of senile gangrene, and case 20, at p. 309, was that of a man, æt. 51, who had vegetations on the aortic valves, with dilated heart's cavities and hypertrophied walls.

The cardiac changes in these three cases are supposed by the authors to be a result of hyalin.-fibroid degeneration of the minute arteries and capillaries. I would suggest, however, that these changes might with more reason be referred in the first case to the emphysematous lungs with obstruction, primarily of the pulmonary, and secondarily of the systemic circulation; in the second case, to degeneration of the walls of the larger arteries resulting in senile gangrene; and in the third case to incompetence of the aortic valves, which is usually deemed sufficient to explain dilatation and hypertrophy of the heart.

In further proof of their doctrine that the arteriocapillary change is the primary and essential condition they refer again, at p. 286, to cases in which the kidneys were healthy while the heart was much hypertrophied.

Three cases are given in illustration of the co-existence of cardiac hypertrophy with healthy kidneys. Case 2, at p. 297, was that of a woman sixty-three years of age, who, therefore, may probably have had senile degeneration of her large arteries. The heart was heavy, but, death having resulted from rupture of the left ventricle, its muscular tissue must have undergone other changes than hypertrophy; and kidneys weighing fifteen ounces could scarcely have been healthy, even though their surface was "almost smooth." Case 3 , at p. 297, was a man, seventy-seven years of age, whose hypertrophied and dilated left ventricle may probably be referred to senile changes in the larger arteries. And, lastly, case 19, at p. 308, was that of a man, aged sixty-two, who died suddenly comatose. His cerebral arteries were very atheromatous, and his lungs were emphysematous. In the condition of the larger arteries and lungs, we have, I think, a sufficient explanation of the dilatation and hyper- 
trophy of the left ventricle, but, in addition, we find that the kidneys weighed only eight ounces; yet we are told that "with the exception of the capsule being adherent they were normal." We find then that one case in which the kidneys weighed fifteen ounces and another in which their weight was only eight ounces are set forth as examples of cardiovascular changes with healthy kidneys. This implies that the kidneys of adults may differ in weight in the proportion of nearly two to one and yet be quite sound in structure.

While the authors refer to cases such as these to prove that hypertrophy of the left ventricle occurs unconnected with renal disease, they maintain that there is a constant association between the cardiac hypertrophy and the hyalinfibroid change in the minute arteries and capillaries, and they state that there is a direct relation between the cardiac and the vascular change (see pp. 289 and 290). Yet it would seem that this pathological law is not without exceptions, for we find it stated at p. 292, par. 2, that " in a few cases the vessels of the pia mater were thickened by this 'hyalinfibroid' change whilst the kidneys and heart were healthy."

Their explanation of the manner in which the vascular change causes the cardiac hypertrophy is the following (p. 290, par. 3) :-“ The hyalin-fibroid material in the walls of the arterioles must be an impediment to elasticity, and it can be experimentally shown that greater force is required to propel a fluid continuously through a non-elastic than through an elastic tube. The left ventricle, therefore, owing to this diminished elasticity of the arterial walls has, of necessity, to contract with greater force to carry on the circulation."

Now, upon this statement I beg to remark, that the explanation of the hypertrophy of the left ventricle is clearly a physiological one; the hypertrophy is said to be a result of the necessity for more forcible contraction to carry on the circulation. Yet the authors, at p. 285, referring to my explanation of the changes in the heart and arterioles as a physiological result, express their belief that "the cardiovascular changes are throughout a morbid one." It appears, 
however, that the authors of this criticism agree with me that the hypertrophy of the left ventricle is a physiological result of excessive muscular contraction called forth by some impediment in the course of the circulation; we differ only as to the cause of the impediment; and I submit that the explanation which I just now quoted from their paper is inconsistent with the facts of anatomy and with the doctrines of physiology.

In that explanation they appear to confound the elasticity of the larger arteries with the muscularity of the smaller vessels. The elastic resiliency of the larger arteries assists in propelling the blood onwards, and converts the intermitting jet from the heart into a continuous stream in the minute arteries and capillaries. The loss of this elasticity, therefore, by degeneration of the arterial walls, involves a loss of propulsive power, and thus imposes extra work on the heart. Hence, the common occurrence of hypertrophy of the left ventricle as a result of senile degeneration of the large arteries. On the other hand, the muscular contractility of the minutest arteries is a force which antagonises the heart and the larger elastic arteries ; so that atrophy and degeneration of the muscular walls of the minute arteries tends to impair not the propelling but the retarding forces concerned in the circulation; and such degenerative changes would involve not an increase but a decrease of resistance to the blood-stream ; unless the minute arteries and capillaries had their walls converted into rigid tubes with constricted canals, of which changes in the minute vessels I shall presently show that we have no proof.

The authors state, at p. 295, vol. lv, that the "hyalinfibroid" change in the arterioles is attended with "atrophy of the adjacent textures in whatever organ it occurs." But I find it difficult to reconcile this statement with another statement which appears at, p. 287 , par. 2 , to the effect that "the minute arteries in the walls of the heart have been found much thickened by the formation of the 'hyalinfibroid 'substance ;" and yet the walls of the heart are not atrophied but hypertrophied. Again the doctrine that 
atrophy of adjacent tissues is an invariable result of thickening of the arterial walls is inconsistent with the fact that this thickening occurs in the minute renal arteries, not only in the small granular kidney, but also in the large smooth kidney of chronic Bright's disease. Obviously, then, thickening of the arterial walls is not, as the authors assert, invariably associated with atrophy of the adjacent tissues.

I now proceed to discuss the nature of those changes in the walls of the blood-vessels to which the authors of the paper give the name of "arterio-capillary fibrosis." When their paper was read before the Society, after hearing the description of the appearances in the walls of the vessels and examining the drawings, but before seeing their specimens, I ventured to suggest that the so-called "hyalin-fibroid" change was simply a result of the external fibrous coat of the artery being distended and rendered translucent by the fluid in which the specimens were preserved, that fluid being, as stated by the authors, a mixture of glycerine and camphor water (p. 280).

An examination of their specimens confirmed my first impression, and my subsequent inquiries have thoroughly convinced me that the hyalin-fibroid appearance is not an indication of a pathological change occurring during life, but a post-mortem physical result of the imbition of fluid by the coats of the vessels. I am indebted to the courtesy of Dr. Sutton for the opportunity of examining a number of his specimens, and I learnt from him that they were all mounted in the glycerine fluid by an assistant before they were examined microscopically by any one.

The authors, therefore, up to the time of reading their paper, had had no opportunity of comparing the appearance of the vessels when recently removed from the body with that which they assume after immersion in glycerine. This comparison I have made in a number of instances, and the following is the result.

1. I have never yet seen the hyaline thickening of the external fibrous tunic in vessels examined immediately after removal from the body. 
2. The appearance is very common, but not constant, after the vessels have been immersed in a mixture of glycerine and camphor water. I have different specimens of arteries from the same subject. Some mounted in the glycerine fluid are conspicuously hyaline, while others preserved in weak spirit and water or in a solution of common salt of sp. gr. 1030 present a perfectly normal appearance. The solution of salt, however, is not sufficiently antiseptic to preserve the specimens for more than a few days.

3. The glycerine fluid produces the hyaline thickening of the minute arteries of the pia mater after death from various diseases, having no relation to Bright's disease, and not being associated with hypertrophy of the left ventricle. Thus, for example, I have specimens of pia mater arteries from a woman who died of diabetes at the age of forty, and whose heart weighed only $6 \frac{3}{4} \mathrm{oz}$. (see figs. 1 and 2). From another

Fig. 1.

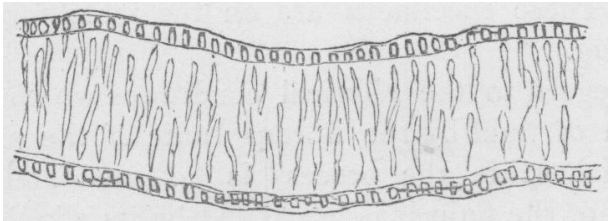

Normal artery from the pia mater, mounted in dilute spirit. $\quad \times 200$.

Fig. 2.

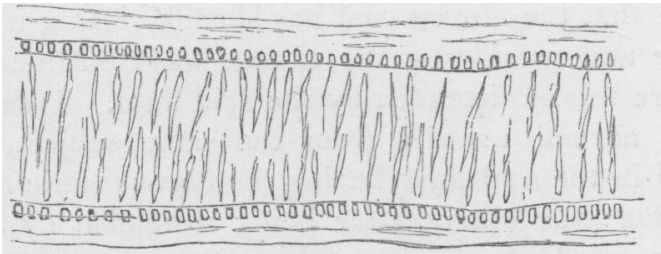

Artery from the same pia mater as No. 1; but the fibrous tissue has been distended and rendered hyaline by the glycerine and camphor water in which it is mounted. $\times 200$.

VOL. LVI. 
woman, æt. 40, who died of cancer of the ovary, whose heart weighed 8 oz. From an infant, aged eleven months, who, having had a cough for a few days, died suddenly, and probably from spasm of the larynx. The kidneys, heart, and all the organs were quite healthy, but the arteries of the pia mater, preserved in the glycerine fluid, present the "hyalinfibroid" change very conspicuously.

I have also specimens of pia mater arteries from a boy, æt. 15, who had been in good health until he was attacked by typhoid fever, during the course of which he died from perforation of the bowel. There was no postmortem evidence of disease except such as resulted from the fever. In particular the heart and kidneys were healthy. Portions of pia mater have been preserved in three different fluids ; 1st, in dilute spirit ; 2nd, in salt and water, sp. gr. 1030 ; and, 3rd, in equal parts of glycerine and camphor water. In Nos. 1 and 2 the vessels appear quite normal; in No. 3 the fibrous tunic is much distended and extremely hyaline. These specimens are on the table for inspection this evening.

With regard to the physical conditions which favour the imbibition of fluid by the fibrous tunic of the arteries it is certain that while the process is favoured and quickened by acidity of the fluid it is prevented by its alkalinity.

The following experiment affords a good illustration of this principle :-Immerse a small piece of pia mater in water, or in glycerine, slightly acidulated by acetic acid; the arteries in a few seconds assume the hyaline appearance; then neutralize the acid by liquid ammonia, and immediately it will be found that the fibrous coat has shrunk, losing its hyaline character and presenting the fibrous wavy outline which it had before it was distended by the acid fluid. I have specimens of normal arteries from the same subject, one set preserved in the acid fluid, having the fibrous tunic distended and hyaline; the others, after being distended by the acid, have been made to shrink and again assume the fibrous character by the addition 'of ammonia; and in that condition they are preserved (see figs. 3 and 4 ). 
FIG. 3:

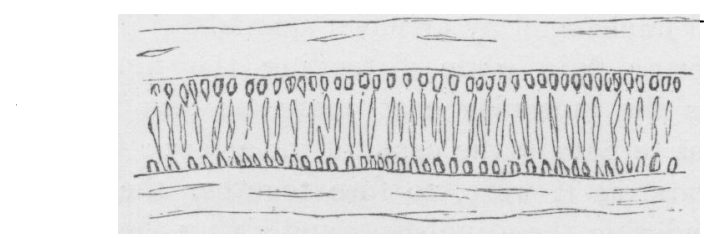

Normal artery from the pia mater, the fibrous tunic distended and rendered hyaline by dilute acetic acid. $>200$.

Fra. 4.

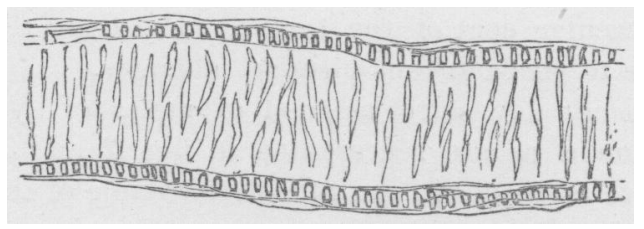

Artery which, after immersion in dilute acetic acid, and thus having been rendered hyaline like No. 3 , has had its fibrous coat again contracted by the addition of ammonia. $\times 200$.

This experiment shows that the appearance of the minute arteries may be rapidly and greatly changed by chemical conditions, which would be influenced by various circumstances, including, amongst others, probably the mode of death and the time after death at which the vessels are examined.

I look upon it as certain that the "hyalin" appearance so often referred to is always the result of post-mortem changes in the arteries. I believe that the appearance in question has never been seen in vessels recently removed from the body, and not subjected to artificial agencies; though it is possible that the change might occur, in the vessels of the pia mater, for instance, as a result of postmortem maceration in the subarachnoid fluid. 
It is incumbent on those who believe that the appearances described are the result of ante-mortem pathological processes to demonstrate them in vessels recently removed from the body and unchanged by artificial agents.

The glycerine fluid while distending the fibrous tunic often presses the muscular coat more or less irregularly inwards, so as to narrow the canal of the artery ; frequently, too, it appears, as it were, to fuse together the muscular elements, rendering them indistinct and giving the thickened wall of the artery a homogeneous or a granular appearance. In other specimens the distension of the arterial wall appears to separate the muscular elements to a greater distance from each other (see Plate VI, fig. 3, in vol. lv). These changes are supposed by the authors of the papers to indicate atrophy of the muscular coat of the artery. I believe them to be simply due to post-mortem physical changes.

I should not now select as good examples of hypertrophy such specimens as are represented in figs. 2 and 3, Plate I, appended to my paper in the fifty-first volume of the 'Transactions.' In these specimens the muscular tissue has been rendered indistinct by the imbibition of glycerine. The appearances here described are quite distinct from the fatty degeneration of the walls of the vessels resulting from senile degeneration, and quite different from the waxy or lardaceous degeneration of the arterial walls.

In some of the arteries the fluid, passing between the middle and inner coat of the vessel, separates them from each other and gives that appearance which the authors describe and figure as a "thickening of the tunica intima." This appearance is shown by the authors in figs. 3 and 4, Plate V, vol. lv.

Again, they affirm that the thickening extends to the walls of the capillaries; it is an "arterio-capillary fibrosis." Here, again, I am compelled to dissent. I have seen no capillaries thickened, except the Malpighian capillaries of the kidney ; and that, in cases of Bright's disease, is probably a result of the continued transudation of albuminous and fibrinous materials through their walls. I have seen no thickening of 
the capillaries in the pia mater. Fig. 2 in Plate VI, which is described as a thickened capillary, $I$ take to be an artery thickened and transformed by glycerine. Fig. 1, in the same plate, with transparent oval bodies in the walls, is unlike any vessel that $I$ have seen, and extremely unlike any capillary of the pia mater.

I do not think it necessary or desirable to criticise at length the author's account of the minute anatomy of the contracted kidney. The sum and substance of their description is that "hyalin-fibroid" material is found here, there, and everywhere in the kidney.

In the year 1847 two papers on renal pathology were read before this Society and published in the thirtieth volume of the 'Transactions.' Mr. Simon was the author of one of these papers and I contributed the other. Both papers were read the same evening; in both papers the same appearances were described and figured, but Mr. Simon and I differed essentially in our interpretation of the phenomenon. The appearances which Mr. Simon described as microscopic cells and cysts $I$ interpreted as tubes denuded and rendered transparent by the destruction and removal of their gland-cells. I have since seen no reason to doubt that my explanation of the appearances in question was the true one, or that the description then given of the minute anatomy of the contracted kidney was, in the main, correct. The appearances which then formed the subject of controversy and which may readily be demonstrated in the contracted Bright's kidney are completely ignored or lightly passed over by those pathologists who make unsatisfactory attempts to explain the structural changes by hyperplasia of connective tissue, or hyalin-fibroid deposit between the tubes.

The description which Sir William Gull and Dr. Sutton give of the appearance presented by the renal arteries in the contracted kidney is so remarkable that it demands especial comment. Fig. 7, in Plate VI, represents a transverse section of a thickened renal artery, and in the description of the plate the artery is said to be "greatly 
thickened by hyalin-fibroid changes in the outer layer of the vessels."

The surface of the section shows two distinct layers of fibres, an inner longitudinal and an outer circular, both of which are muscular, as may be clearly seen in many specimens in my possession. Sir William Gull and Dr. Sutton, however, assume that the inner longitudinal layer alone is muscular, and at p. 274 (par. 6) they say that "external to the muscular nuclei there was a quantity of hyalin-fibroid substance, and the layer formed by this material was much thicker than the muscular layer" (Plate VI, fig. 7, in vol. lv). Again, they say (at p. 278, par. 4), "Where the kidney disease was far advanced hyalin-fibroid changes were seen in the minute renal arteries precisely similar to those observed in the arterioles of the pia mater and of other parts of the body." It is evident, therefore, that they believe the outer layer in the renal artery to be the counterpart of the so-called "hyalin-fibroid" layer in the arteries of the pia mater. The fact being that this layer in the pia mater arteries is external to the circular muscular fibres, and is composed of the distended fibrous tunic; while in the renal artery the fibrous tunic is inconspicuous and that which they mistake for it is the circular layer of muscular fibres.

In my first published account of the hypertrophied renal arteries in cases of chronic Bright's disease ('Med. Chir. Trans.,' vol. xxxiii) I described and figured the inner longitudinal and the onter circular layer of muscular fibres. The appearances described are quite constant; they were well seen in some of the author's specimens exhibited at the conversazione in June, and were clearly represented by their artist; yet they have been misinterpreted in the manner described. The existence of hypertrophy of the muscular walls is denied and the hyalin-fibroid change is supposed to explain the phenomena.

At the foot of page 277 we find, in a note, the following statement :-

"Recent observations on the minute anatomy of the 
kidney raise a doubt whether casts formed in the convoluted tubules* of the cortex can escape."

It would be interesting to know what observations have led to this curious piece of scepticism. No one who has studied the microscopic diagnosis of renal diseases can, for a moment, doubt that casts from the convoluted tubes of the cortex continually escape and are found in the urine. If this were not so the various forms of tube-casts would throw no light upon the state of the cortical portion of the kidneys, which is acknowledged to be the seat of Bright's disease. We find, however, that these casts from the cortex sometimes retain their convoluted form even after passing through the straight tubes. They often contain epithelial cells which, from their characteristic appearance, are known with absolute certainty to have come from the cortex and not from the tubes of the cones. And, lastly, in some cases of hæmaturia, blood which has been poured into the very extremities of the convoluted tubes from ruptured Malpighian capillaries, escapes and appears in the form of blood casts in the urine.

To doubt the possibility of the blood passing from the right to the left side of the heart through the lungs would be scarcely more unreasonable than to doubt the escape of casts from the convoluted tubes of the kidney in most cases of Bright's disease.

The authors object to my doctrine that the hypertrophy of the left ventricle, in cases of chronic Bright's disease, is a consequence of the increased resistance to the circulation occasioned by excessive contraction and resulting hypertrophy of the minute muscular arteries. They say truly that hypertrophy of the heart, although usually present in the advanced stages of the contracted kidney, is absent in a large proportion of cases of large white kidney.

My belief is that when, in cases of chronic Bright's disease with either large or small kidneys, the left ventricle is hypertrophical without disease of the valves or of the

* In the note referred to, "convoluted tubercles" is evidently a misprint for "convoluted tubules." 
large arteries to explain it; in all such cases the minute arteries throughout the body, but especially in the skin and mucous membranes, will be found to have their muscular walls hypertrophied. I have as yet met with no exception to this rule. In explanation of the common occurrence of hypertrophy of the left ventricle in connection with the contracted kidney and its frequent absence in cases of large white kidney, I beg to suggest the following considerations :

1st. With the contracted kidney there is, as a rule, absence of dropsy or but little dropsy, a free secretion of water by the kidney, with retention of urinary solids in the blood; and this uræmic condition is, in various ways, the cause of death. On the other hand, in cases of large white kidney, there is a more scanty secretion of water, and, in consequence, a state of hydræmia and dropsy. In the first class of cases the urinary solids accumulating in the blood probably excite the contraction of the systemic arterioles, and thus directly cause their hypertrophy and indirectly that of the left ventricle. In the second class of cases-those associated with large white kidneys-while there is some retention of urinary solids there is a disproportionate accumulation of water, by which the contaminated blood is diluted and thus rendered less irritating to the minute arteries.

This is probably, in part at least, the explanation of the fact that the arterial resistance is less and the cardiac hypertrophy less constant and pronounced in cases of large white kidney with consequent hydræmia than in cases of contracted kidney with resulting uræmia.

2nd. But there is yet another class of facts to be taken into account. In connection with the lardaceous form of disease in the kidney and in other organs there is usually a widely spread tendency to degeneration of the small arteries. The degenerative changes affecting the muscular walls impair their contractile power, and the result is not an increase but a decrease of resistance in the terminal arteries. The peripheral resistance which calls forth increased contraction with resulting hypertrophy of the left ventricle is wanting, and in these circumstances there is absence of cardiac 
hypertrophy. It appears to me that these apparently exceptional cases prove the rule; namely, that there is, in the absence of valvular lesions and senile degeneration of the walls of the large arteries, a direct and constant relation between hypertrophy of the left ventricle of the heart and hypertrophy of the muscular walls of the minute arteries throughout the body. The occasional, though rare, occurrence of fatal cases of contracted kidney without cardiac hypertrophy is not inconsistent with the theory that when they coexist they stand to each other in the relation of cause and effect. Cases of large white kidney sometimes run their course without the occurrence of dropsy; but we do not, therefore, deny or doubt that the dropsy which is frequently associated with this form of disease is a result of the blood changes induced by the renal disease.

It is stated at p. 289 of the author's paper " that in eighteen cases the left ventricle was found hypertrophied without valvular disease or pericardial adhesion to explain it, while the kidneys were healthy excepting that they were slightly granular, or, in other words, 'coarse' and contained some cysts." It is therefore inferred that in these eighteen cases the hypertrophy was "the older and preceding condition." Upon this statement I would suggest-lst. That a minute examination of the kidneys might have shown more advanced degeneration than appeared upon the surface; and, 2nd, it is not unlikely that in some of these cases the cardiac hypertrophy was, in part at least, explained by degeneration of the walls of the larger arteries, as in some of their cases occurring in old people to which I have before referred.

The atheromatous degeneration of the large arteries, which is often associated with chronic Bright's disease, may be in part, perhaps, explained by the noxious influence of contaminated blood, and in part by the undue strain to which the arteries are subjected between the two opposed forces, that of the hypertrophied left ventricle behind and that of the resisting muscular arterioles in front.

I need not here repeat my objection to the author's 
doctrine that hypertrophy of the left ventricle is always associated with and explained by the hyalin-fibroid change in the minute arteries; this change being, as $I$ believe, not a pathological change at all but the result of artificial influences operating after death.

The statement that the morbid changes included under the head of chronic Bright's disease with contracted kidney are the result of "causes not yet ascertained" is not in accordance with the results of my own observation.

There are few cases of this form of disease which are not traceable to some probable cause. Amongst the most common causes is the excessive consumption of food and of alcoholic stimulants either with or without the association of decided gouty symptoms; but I have seen many cases in which, as $I$ believe, this form of renal disease has resulted from chronic dyspepsia in persons of strictly temperate habits. The one condition which is common to all these cases is that the degeneration of the kidney is directly caused by the excretion of abnormal and irritating materials, the products of faulty digestion ; more commonly associated with habitual excess of food and stimulants, but not rarely without such excess.

I have always maintained that Bright's disease, in all its forms, may be traced back to some anterior morbid change in other tissues and organs, the proximate cause of the renal disease in every case being the contamination of the circulating blood by abnormal products, which the kidney, in the discharge of its excretory function, is called upon to eliminate.

The doctrine that the area of the morbid state under discussion "may be said hypothetically to correspond to the area vasculosa" is too transcendental for everyday use, and it is not in accordance with the fact that the morbid changes are, as a rule, limited to definite portions of the arterial system, while the veins and capillaries, excepting only the Malpighian capillaries, are not implicated. The term " arterio-capillary fibrosis" appears to be a misnomer, for the capillaries generally are unchanged, and if my interpre- 
tation of the phenomena is correct there is no morbid "fibrosis" of the arteries.

In conclusion, I beg to express my thanks to Sir William Gull and Dr. Sutton for having so forcibly directed attention to the cardio-vascular changes in chronic Bright's disease. Differing as we do entirely in our interpretation of the phenomena we agree in the opinion that the subject is one of great interest and importance. 